\title{
The Derived Category of quasi-coherent sheaves on an Artin stack via model structures
}

\author{
Sergio Estrada* \\ sestrada@um.es \\ Departamento de Matemática Aplicada \\ Universidad de Murcia \\ 30100 Murcia \\ SPAIN
}

\begin{abstract}
We define the derived category of quasi-coherent modules for certain Artin stacks as the homotopy category of two Quillen monoidal model structures on the corresponding category of unbounded complexes of quasi-coherent modules.
\end{abstract}

2010 Mathematics Subject Classification. Primary 14A20; Secondary 55U35,18E30

Key words and phrases: algebraic stack, Deligne-Mumford stack, cartesian R-module, Grothendieck category, model category structure.

\section{Introduction}

In EE05] we develop a method for finding a family of generators of the so-called category of quasicoherent $R$-modules on an arbitrary quiver (cf. [EE05, Corollary 3.5]) and we prove that the class of flat quasi-coherent $R$-modules is covering (cf. [EE05, Theorem 4.1]). The first part of the present paper is devoted to showing that the same arguments of [EE05] can also be used in a much more general setup, that is that of cartesian $R$-modules on a flat presheaf of rings $R$ over a small category $\mathcal{C}$. This extends the main application in EE05] to the category $\mathfrak{Q} \mathfrak{c o}(X)$ of quasi-coherent sheaves on a scheme $X$ but also to the category $\mathfrak{Q} \mathfrak{c o}(\mathcal{X})$ of quasi-coherent sheaves on an Artin stack or on a Deligne-Mumford stack. This seems to be known to some authors, but the lack of a published result of this fact in the literature becomes it into an interesting consequence of the results of EE05.

\footnotetext{
*This paper has been completed during the author's stay at Max Planck Institute for Mathematics in Bonn. The author would like to thank its hospitality and the excellent conditions provided for his stay at the institution.
} 
In the second part of the paper we deal with the derived category of quasi-coherent sheaves on an Artin stack. From its abstract definition one has little control over the morphisms in the derived category and in fact it is not clear if we have only a set of maps between any two objects. A solution to these questions is provided by defining a good Quillen's theory of model categories in $\operatorname{Ch}(\mathfrak{Q} \mathfrak{c o}(\mathcal{X})$ ) (Qui67) where the weak equivalences are the quasi-isomorphisms. Then it follows from Quillen's theory that the corresponding homotopy categoy, the derived category of $\mathfrak{Q} \mathfrak{c o}(\mathcal{X})$, is truly a category. Moreover there is a simple description of the set of morphisms from two objects $M$ and $N$ in the derived category as chain homotopy classes of chain maps from a cofibrant replacement for $M$ to a fibrant replacemnent of $N$. Moreover, the category $\mathfrak{Q} \mathfrak{c o}(\mathcal{X})$ has a tensor product, which naturally inherits to $\operatorname{Ch}(\mathfrak{Q} \mathfrak{c o}(\mathcal{X}))$, for which we should be able to compute left derived functors, so we would like that our Quillen model category structures to be monoidal. To achieve this goal, we give a general theorem (Theorem 7.2) that guarantees the existence of cofibrantly generated model category structures in the category of unbounded complexes of cartesian $R$-modules and later in Section 8 we specialize to the category of quasi-coherent $\mathcal{O}_{X}$-modules over Artin stacks. This is an extension of the previous papers Gil07 and EGPT12 from schemes to algebraic stacks. Our main application is that for a geometric stack $\mathcal{X}$ (see [TV08] and [Lur05]) we show the existence of a flat monoidal model category structure on $\operatorname{Ch}(\mathfrak{Q} \mathfrak{c o}(\mathcal{X}))$ (Theorem 8.1) and for algebraic stacks that satisfy the resolution property (these include global quotient stacks) we show in Theorem 8.2 that there is a locally projective monoidal model structure on $\operatorname{Ch}(\mathfrak{Q} \mathfrak{c o}(\mathcal{X}))$. One immediate implication from May01 is then that the triangulated structure of $\mathbf{D}(\mathfrak{Q} \mathfrak{c o}(\mathcal{X}))$ is strongly compatible with the derived tensor product. We finish Section 8 with a list of consequences of the results of this paper for categories of modules over a flat Hopf algebroid and on the existence of adjoint functors in homotopy categories for algebraic stacks.

\section{Cartesian modules on quivers}

A quiver $Q$ is a directed graph. An edge of a quiver from a vertex $v_{1}$ to a vertex $v_{2}$ is denoted by $a: v_{1} \rightarrow v_{2}$ or $v_{1} \stackrel{a}{\rightarrow} v_{2}$, the symbol $E$ will denote the set of edges. A quiver $Q$ may be thought as a 
category in which the objects are the vertices of $Q$ and the morphisms are the paths (a path is a sequence of edges) of $Q$. The set of all vertices will be denoted by $V$.

Let $Q=(V, E)$ be a quiver and let $R$ be a presheaf from $Q$ in the category of commutative rings, that is, for each vertex $v \in V$ we have a ring $R(v)$ and for an edge $a: v \rightarrow w$ we have a ring homomorphism $R\left(a^{o p}\right): R(w) \rightarrow R(v)$.

We shall say that we have an $R$-module $M$ when we have an $R(v)$-module $M(v)$ and a morphism $M\left(a^{o p}\right): M(w) \rightarrow M(v)$ for each edge $a: v \rightarrow w$ that is $R(v)$-linear. The $R$-module $M$ is said to be a cartesian $Q$-module if for each edge $a: v \rightarrow w$ as above the morphism

$$
R(v) \otimes_{R(w)} M(w) \rightarrow M(v)
$$

given by $r_{v} \otimes m_{w} \mapsto r_{v} M\left(a^{o p}\right)\left(m_{w}\right), r_{v} \in R(v), m_{w} \in M(w)$ is an $R(v)$-isomorphism.

The category of cartesian $Q$-modules is abelian when $R$ is such that for an edge $v \rightarrow w, R(v)$ is a flat $R(w)$-module (so the kernel of a morphism between two cartesian $Q$-modules is also cartesian). In this case we say $R$ is flat. Coproducts and colimits may be computed componentwise so direct limits are exact and, as a result of Proposition 4.2. we can find a system of generators in the category. Therefore the category of cartesian $Q$-modules is indeed a Grothendieck category when $R$ is flat.

By the tensor product, $M \otimes_{R} N$, where $M$ is a right $R$-module and $N$ a left $R$-module, we mean the $\mathbb{Z}$-module $\left(\mathbb{Z}(v)=\mathbb{Z}\right.$, for all $v \in V$ and $\mathbb{Z}(a)=i d_{\mathbb{Z}}$ for all $\left.a \in E\right)$ such that

$$
\left(M \otimes_{R} N\right)(v)=M(v) \otimes_{R(v)} N(v),
$$

with $\left(M \otimes_{R} N\right)(a)$ the obvious map. We then get the notion of a flat R-module and a flat cartesian $Q$-module. We also get the notion of locally projective cartesian $Q$-module $M$, by defining that $M(v)$ is a projective $R(v)$-module for each $v \in V$.

Given an arbitrary quiver $Q$ and a flat presheaf of $\operatorname{rings} R$ over $Q$, we will denote by $\operatorname{QMod}_{\mathrm{cart}}(R)$ the category of cartesian $Q$-modules over $R$. 


\section{Cartesian modules on small categories}

Now let $\mathcal{C}$ be any small category, and let $R$ be a flat presheaf of rings on $\mathcal{C}$. We will consider the category $\operatorname{Mod}_{\text {cart }}(R)$ of cartesian $R$-modules. This is an abelian category and, as a consequence of Proposition 4.2 . it will be a Grothendieck category. There is a notion of flat and of locally projective cartesian module as before. Let $Q$ be the quiver whose vertices are the objects of $\mathcal{C}$, and whose edges are the morphisms of $\mathcal{C}$. It is then clear that the category $\operatorname{Mod}_{\text {cart }}(R)$ is a full subcategory of the category $Q M o d_{\text {cart }}(R)$. Furthermore it is also clear that if $M \subseteq N$ in $\operatorname{QMod}_{\text {cart }}(R)$ and $N$ is a cartesian $R$-module, then $M$ will be automatically a cartesian $R$-module as well. This easy observation is crucial in proving our main result in the next section and giving our main applications.

\section{Generators in $\operatorname{Mod}_{\text {cart }}(R)$. Application to algebraic stacks}

With the observations made in the previous sections, we can use Proposition 3.3 of EE05] to infer that $\operatorname{Mod}_{\text {cart }}(R)$ is a Grothendieck category. Throughout this section we will assume that $\mathcal{C}$ is a small category and $R$ is a flat presheaf of rings on it. We shall denote by $Q$ the quiver associated to $\mathcal{C}$.

Definition 4.1. Let $M$ be a cartesian $Q$-module. The cardinality of $M$ is defined as the cardinality of the coproduct (in the category of sets) of all modules associated to the vertices $v \in V$, that is

$$
|M|=\left|\sqcup_{v \in V} M(v)\right|
$$

Proposition 4.2. Let $\mathcal{C}$ be any small category with associated quiver $Q_{\mathcal{C}}=(V, E)$ and $M$ a cartesian $R$-module. Let $\lambda$ be an infinite cardinal such that $\lambda \geq|R(v)|$ for all $v$ and such that $\lambda \geq \max \{|E|,|V|\}$. Let $X_{v} \subseteq M(v)$ be subsets with $\left|X_{v}\right| \leq \lambda$ for all $v$. Then there is cartesian $R$-submodule $M^{\prime} \subseteq M$ with $M^{\prime}(v)$ pure for all $v$, with $X_{v} \subseteq M^{\prime}(v)$ for all $v$ and such that $\left|M^{\prime}\right| \leq \lambda$.

Proof. The proof of [EE05, Proposition 3.3] gives a cartesian $Q$-submodule $M^{\prime}$ of $M$ satisfying the desired properties. But then by the previous comment, as $M$ is cartesian, $M^{\prime}$ will be also cartesian $R$-module. $\square$. 
Definition 4.3. A cartesian $R$-submodule $M^{\prime}$ of an $R$-module $M$ is said to be pure whenever $M^{\prime}(v)$ is a pure $R(v)$-submodule of $M(v)$, for every vertex $v \in V$.

Corollary 4.4. There exists an infinite cardinal $\lambda$ such that every cartesian $R$-module $M$ is the sum of its quasi-coherent $R$-submodules of type $\lambda$.

Proof. Let $M$ be any cartesian $R$-module and take an element $x \in M$. Then, by Proposition 4.2 we find a (pure) cartesian $R$-submodule $S_{x}$ of $M$ with $\left|S_{x}\right| \leq \lambda$ and $x \in S_{x}$. Thus $M=\sum_{x \in M} S_{x}$.

As a consequence of this we have that $\operatorname{Mod}_{\text {cart }}(R)$ is a Grothendieck category whenever $R$ is a flat presheaf of rings, for if we take a set $Z$ of representatives of cartesian modules with cardinality bounded by $\lambda$, it is immediate that the single cartesian $R$-module $\oplus_{S \in Z} S$ generates the category of quasi-coherent $R$-modules.

Now if we focus on particular instances of small categories we have the following significant consequences. The first one is due to Gabber (cf. Con00, Lemma 2.1.7]).

Corollary 4.5. Let $\left(X, \mathcal{O}_{X}\right)$ be any arbitrary scheme. Each quasi-coherent sheaf can be written as a sum of its pure quasi-coherent subsheaves of type $\lambda$. Thus $\mathfrak{Q} \mathfrak{c o}(X)$ is a Grothendieck category.

Proof. We let $\mathcal{C}$ consisting of all the affine open $U \subseteq X$. Then the inclusion between affine open subsets defines a canonical structure of a partially ordered category on $\mathcal{C}$. Now we let $R$ be the structure sheaf $\mathcal{O}_{X}$. Then it is standard that $\operatorname{Mod}_{\text {cart }}(R)$ and $\mathfrak{Q} \mathfrak{c o}(X)$ are equivalent categories. So the result will follow from Corollary 4.4

Remark. The previous proof also clarifies a possible misunderstanding on [EE05, Section 2]. There, the reader may wrongly think that we are considering the free category on the affine open subsets of the scheme $X$ to establish our equivalent category $\mathcal{C}$. This is obviously not true, and the gap is easily fixed by saying that we were assuming the compatibility condition on our representations there to get the desired equivalence. To be precise we are just claiming that $\mathfrak{Q} \mathfrak{c o}(X)$ and $\operatorname{Mod}_{\text {cart }}(R)$ (or $\mathcal{C}$ in that section) are equivalent. 
Our second application goes back to Artin stacks (cf. [Ols07]) and Deligne-Mumford stacks.

Corollary 4.6. Let $\mathcal{X}$ be a Deligne-Mumford stack. Then the category $\mathfrak{Q} \mathfrak{c o}(\mathcal{X})$ is a Grothendieck category. In particular it is locally presentable and has arbitrary products.

Proof. We take $\mathcal{C}$ as the small subcategory of the iso classes of the category of affine schemes that are étale over $\mathcal{X}$ (such small subcategory must exist as the iso classes of such schemes form a set, as étale morphisms are of finite type). Then $\operatorname{Mod}_{\text {cart }}(R)$ is equivalent to $\mathfrak{Q} \mathfrak{c o}(\mathcal{X})$.

Corollary 4.7. Let $\mathcal{X}$ be an algebraic stack with a flat sheaf of rings $\mathcal{A}$. Then the category $\mathfrak{Q} \mathfrak{c o}(\mathcal{X})$ is a Grothendieck category. In particular it is locally presentable and has arbitrary products.

Proof. In this case we consider $\mathcal{C}$ to be the category of affine schemes smooth over $\mathcal{X}$ and $R$ as the sheaf of rings $\mathcal{A}$. Then $\operatorname{Mod}_{\text {cart }}(R)$ is equivalent to the category of quasi-coherent sheaves on $\mathcal{X}$.

\section{$5 \quad$ Preliminaries on cotorsion pairs in $\operatorname{Mod}_{\text {cart }}(R)$}

In this section we present those notions from $\operatorname{Mod}_{\text {cart }}(R)$ which will be used in the sequel. Let $\mathcal{C}$ be any small category, $R$ a flat presheaf of rings on $\mathcal{C}$ and $\operatorname{Mod}_{\text {cart }}(R)$ the corresponding category of cartesian $R$-modules. Let us fix $\lambda$ as in Proposition 4.2

We recall that a cartesian $R$-module $M$ is $\kappa$-generated (for $\kappa$ a regular cardinal) whenever

$$
\operatorname{Hom}_{M_{\text {cart }}(R)}(M,-)
$$

preserves $\kappa$-filtered colimits of monomorphisms. Equivalently, $M$ is $\kappa$-generated whenever $M=\sum_{i \in I} M_{i}$ is a $\kappa$-directed union of cartesian submodules, we have $M=M_{i}$ for some $i \in I$. And $M$ is $\kappa$-presentable whenever $\operatorname{Hom}_{M o d} \operatorname{cart}_{\text {cart }}(M,-)$ preserves $\kappa$-filtered colimits. Under our above assumption on $\lambda$ relative to $\operatorname{Mod}_{\text {cart }}(R)$, a cartesian $R$-module $M$ is $\lambda$-presentable if and only if $M$ is $\lambda$-generated and every epimorphism $L \rightarrow M$ with $L \lambda$-generated has a $\lambda$-generated kernel.

Furthermore, given $M \in \operatorname{Mod}_{\text {cart }}(R)$ it is easy to check that the following conditions are equivalent:

1. $|M|<\lambda$. 
2. $M$ is $\lambda$-generated.

3. $M$ is $\lambda$-presentable.

A well-ordered direct system of cartesian $R$-modules, $\left(A_{\alpha} \mid \alpha \leq \gamma\right)$, is said to be continuous if $A_{0}=0$ and, for each limit ordinal $\beta \leq \gamma$, we have $A_{\beta}=\lim _{\rightarrow} A_{\alpha}$ where the limit is taken over all ordinals $\alpha<\beta$. A continuous direct system $\left(A_{\alpha} \mid \alpha \leq \gamma\right)$ is called a continuous directed union if all morphisms in the system are monomorphisms.

Definition 5.1. Let $\mathcal{L}$ be a class of cartesian $R$-modules. An object $A$ of $\mathcal{A}$ is $\mathcal{L}$-filtered if $A=\underset{\rightarrow}{\lim } A_{\alpha}$ for a continuous directed union $\left(A_{\alpha} \mid \alpha \leq \gamma\right)$ satisfying that, for each $\alpha+1 \leq \gamma, \operatorname{Coker}\left(A_{\alpha} \rightarrow A_{\alpha+1}\right)$ is isomorphic to an element of $\mathcal{L}$.

We can easily extend Hill's Lemma (see GT06, Theorem 4.2.6]) to $\operatorname{Mod}_{\text {cart }}(R)$. Let $\mathcal{J}$ be a class of $\lambda$-presentable cartesian $R$-modules and let $M$ be a cartesian $R$-module possesing a $\mathcal{J}$-filtration $\mathcal{O}=$ $\left(M_{\alpha} \mid \alpha \leq \sigma\right)$.

By Proposition 4.2, there exist $\lambda$-presentable cartesian $R$-submodules $A_{\alpha} \subseteq M_{\alpha+1}$ such that $M_{\alpha+1}=$ $M_{\alpha}+A_{\alpha}$ for each $\alpha<\sigma$. A set $S \subseteq \sigma$ is called closed provided that $M_{\alpha} \cap A_{\alpha} \subseteq \sum_{\beta<\alpha, \beta \in S} A_{\beta}$ for each $\alpha \in S$.

Lemma 5.2. Let $\mathcal{H}=\left\{\sum_{\alpha \in S} A_{\alpha} \mid S\right.$ closed $\}$. Then $\mathcal{H}$ satisfies the following conditions:

$(H 1) \mathcal{O} \subseteq \mathcal{H}$

(H2) $\mathcal{H}$ is closed under arbitrary sums,

(H3) $P / N$ has a $\mathcal{J}$-filtration whenever $N, P \in \mathcal{H}$ are such that $N \subseteq P$.

(H4) If $N \in \mathcal{H}$ and $X$ is a $\lambda$-presentable cartesian $R$-submodules of $M$, then there exists $P \in \mathcal{H}$ such that $N+X \subseteq P$ and $P / N$ is $\lambda$-presentable.

Proof. Note that for each ordinal $\alpha \leq \sigma$, we have $M_{\alpha}=\sum_{\beta<\alpha} A_{\beta}$, hence $\alpha$ is a closed subset of $\sigma$. This proves condition (1). Since any union of closed subsets is closed, condition (2) holds. 
In order to prove condition (3), we consider closed subsets $S, T$ of $\sigma$ such that $N=\sum_{\alpha \in S} A_{\alpha}$ and $P=\sum_{\alpha \in T} A_{\alpha}$. Since $S \cup T$ is closed, we will w.l.o.g. assume that $S \subseteq T$. We define a $\mathcal{J}$-filtration of $P / N$ as follows. For each $\beta \leq \sigma$, let $F_{\beta}=\left(\sum_{\alpha \in T \backslash S, \alpha<\beta} A_{\alpha}+N\right) / N$. Then $F_{\beta+1}=F_{\beta}+\left(A_{\beta}+N\right) / N$ for $\beta \in T \backslash S$ and $F_{\beta+1}=F_{\beta}$ otherwise.

Let $\beta \in T \backslash S$. Then $F_{\beta+1} / F_{\beta} \cong A_{\beta} /\left(A_{\beta} \cap\left(\sum_{\alpha \in T \backslash S, \alpha<\beta} A_{\alpha}+N\right)\right)$, and since $\beta \in T \backslash S$ and $T$ is closed, we have

$$
\begin{gathered}
A_{\beta} \cap\left(\sum_{\alpha \in T \backslash S, \alpha<\beta} A_{\alpha}+N\right)=A_{\beta} \cap\left(\sum_{\alpha \in S, \alpha>\beta} A_{\alpha}+\sum_{\alpha \in T, \alpha<\beta} A_{\alpha}\right) \supseteq \\
\supseteq A_{\beta} \cap\left(\sum_{\alpha \in S, \alpha>\beta} A_{\alpha}+\left(M_{\beta} \cap A_{\beta}\right)\right) \supseteq M_{\beta} \cap A_{\beta} .
\end{gathered}
$$

Let $B_{\beta}=\sum_{\alpha \in S, \alpha>\beta} A_{\alpha}+\sum_{\alpha \in T, \alpha<\beta} A_{\alpha}$ We will prove that $A_{\beta} \cap B_{\beta}=M_{\beta} \cap A_{\beta}$. We have only to show that, $A_{\beta} \cap B_{\beta} \subseteq A_{\beta} \cap M_{\beta}$. Let $a \in A_{\beta} \cap B_{\beta}$. Then $a=c+a_{\alpha_{0}}+\cdots+a_{\alpha_{k}}$ where $c \in \sum_{\alpha \in T, \alpha<\beta} A_{\alpha} \subseteq M_{\beta}$, $\alpha_{i} \in S$ and $a_{\alpha_{i}} \in A_{\alpha_{i}}$ for all $i \leq k$ and $\alpha_{i}>\alpha_{i+1}$ for all $i<k$. W.l.o.g., we can assume that $\alpha_{0}$ is minimal possible. If $\alpha_{0}>\beta$, then $a_{\alpha_{0}}=a-c-a_{\alpha_{1}}+\cdots-a_{\alpha_{k}} \in M_{\alpha_{0}} \cap A_{\alpha_{0}} \subseteq \sum_{\alpha \in S, \alpha<\alpha_{0}} A_{\alpha}$ (since $\alpha_{0} \in S$ ), in contradiction with the minimality of $\alpha_{0}$. Since $\beta \notin S$, we infer that $\alpha_{0}<\beta, a \in M_{\beta}$, and $A_{\beta} \cap B_{\beta}=A_{\beta} \cap M_{\beta}$.

So if $\beta \in T \backslash S$ then $F_{\beta+1} / F_{\beta} \cong A_{\beta} /\left(M_{\beta} \cap A_{\beta}\right) \cong M_{\beta+1} / M_{\beta}$, and the latter is isomorphic to an element of $\mathcal{J}$ because $\mathcal{O}$ is a $\mathcal{J}$-filtration of $M$. This finishes the proof of condition (3).

For condition (4) we first claim that each subset of $\sigma$ of cardinality $<\lambda$ is contained in a closed subset of cardinality $<\lambda$. Since $\lambda$ is regular and unions of closed sets are closed, it suffices to prove the claim only for one-element subsets of $\sigma$. By induction on $\beta$ we prove that each $\beta<\sigma$ is contained in a closed set $S$ of cardinality $<\lambda$. If $\beta<\lambda$ we take $S=\beta+1$.

Otherwise, consider the short exact sequence $0 \rightarrow M_{\beta} \cap A_{\beta} \rightarrow A_{\beta} \rightarrow M_{\beta+1} / M_{\beta} \rightarrow 0$. By our assumption on $\lambda$, since $A_{\beta}$ is $\lambda$-presentable, so is $M_{\beta} \cap A_{\beta}$. Hence, $M_{\beta} \cap A_{\beta} \subseteq \sum_{\alpha \in S} A_{\alpha}$ for a subset $S \subseteq \beta$ of cardinality $<\lambda$. By our inductive premise, the set $S$ is contained in a closed subset $S^{\prime}$ of cardinality $<\lambda$. Let $S^{\prime \prime}=S^{\prime} \cup\{\beta\}$. Then $S^{\prime \prime}$ is closed because $S^{\prime}$ is closed, and $M_{\beta} \cap A_{\beta} \subseteq \sum_{\alpha \in S^{\prime}} A_{\alpha}$.

Finally if $N=\sum_{\alpha \in S^{\prime \prime}} A_{\alpha}$ and $X$ is a $\lambda$-presentable cartesian submodule of $M$, then $X \subseteq \sum_{\alpha \in T} A_{\alpha}$ for a subset $T$ of $\sigma$ of cardinality $<\lambda$. By the above we can assume that $T$ is closed and put $P=\sum_{\alpha \in S^{\prime \prime} \cup T} A_{\alpha}$. 
By (the proof of) condition (3) $P / N$ is $\mathcal{J}$-filtered, and the length of the filtration can be taken $\leq|T \backslash S|<\lambda$. This implies that $P / N$ is $\lambda$-presentable.

A cotorsion pair in a Grothendieck category $\mathcal{A}$ is a pair of classes of objects of $\mathcal{A},(\mathcal{F}, \mathcal{C})$, such that $\mathcal{F}={ }^{\perp} \mathcal{C}$ and $\mathcal{C}=\mathcal{F}^{\perp}$, where

$$
{ }^{\perp} \mathcal{C}=\left\{F \in \mathcal{A}: \operatorname{Ext}_{\mathcal{A}}^{1}(F, D)=0, \forall D \in C\right\}
$$

and

$$
\mathcal{F}^{\perp}=\left\{C \in \mathcal{A}: \operatorname{Ext}_{\mathcal{A}}^{1}(G, C)=0, \forall G \in \mathcal{F}\right\} .
$$

The cotorsion pair is said to be cogenerated by a class of objects $\mathcal{T}$ in $\mathcal{A}$ if $\mathcal{T}^{\perp}=\mathcal{C}$. When this class $\mathcal{T}$ is a set and it contains a generator of $\mathcal{A}$, it is known that every object $M$ in $\mathcal{A}$ has enough projectives, that is, there exists a short exact sequence

$$
0 \rightarrow C \rightarrow F \rightarrow M \rightarrow 0
$$

with $F \in \mathcal{F}$ and $C \in \mathcal{C}$, and enough injectives, that is, an exact sequence

$$
0 \rightarrow M \rightarrow C^{\prime} \rightarrow F^{\prime} \rightarrow 0
$$

with $F^{\prime} \in \mathcal{F}$ and $C^{\prime} \in \mathcal{C}$ (see e.g. [EEGRO04, Theorem 2.5] or [Hov02, Corollary 6.6]). A cotorsion pair having enough injectives and projectives is called complete.

By using the previous version of Hill's Lemma, we can get the analogous version of Kaplansky's Theorem for cotorsion pairs (see GT06, Theorem 4.2.11]) for $\operatorname{Mod}_{\text {cart }}(R)$. Given a class $\mathcal{F}$ in $\operatorname{Mod}_{\text {cart }}(R)$ we will denote by $\mathcal{F}^{\kappa}$ the class of all $\kappa$-presentable objects in $\mathcal{F}$.

Theorem 5.3. Let $\kappa$ be an uncountable regular cardinal such that $\kappa \geq \lambda$. Let $(\mathcal{F}, \mathcal{C})$ be a cotorsion pair in $\operatorname{Mod}_{\text {cart }}(R)$ such that $\mathcal{F}$ contains a set of $\kappa$-presentable generators of $\operatorname{Mod}_{\text {cart }}(R)$. Then the following conditions are equivalent:

1. the cotorsion pair $(\mathcal{F}, \mathcal{C})$ is cogenerated by a class of $\kappa$-presentable cartesian $R$-modules.

2. Every cartesian $R$-module in $\mathcal{F}$ is $\mathcal{F}^{\kappa}$-filtered. 
Proof. (1) $\Rightarrow(2)$ We can assume that $(\mathcal{F}, \mathcal{C})$ is cogenerated by a set $\mathcal{T}$ of $\kappa$-presentable cartesian $R$ modules and that, up to isomorphism, each generator of $\operatorname{Mod}_{\text {cart }}(R)$ is in $\mathcal{T}$. By the arguments given in the proof of EEGRO04, Lemma 2.4 and Theorem 2.5], the class $\mathcal{F}$ consists of all retractions of $\mathcal{T}$-filtered objects. So the claim follows with the same proof (adapted to our setting and by using Lemma 5.2) of GT06, Lemma 4.2.10].

$(2) \Rightarrow(1)$ By Eklof's Lemma (Ekl77, Theorem 1.2]) in his version for arbitrary Grothendieck categories, an object $D \in \mathcal{C}$ if, and only if, $D \in\left(\mathcal{F}^{\kappa}\right)^{\perp}$. So $(\mathcal{F}, \mathcal{C})$ is cogenerated by $\mathcal{F}^{\kappa}$.

Remark: Theorem 5.3 can be easily extended to $\mathrm{Ch}\left(\operatorname{Mod}_{\mathrm{cart}}(R)\right)$, the category of unbounded chain complexes of cartesian $R$-modules.

\section{Complete cotorsion pairs in $\mathrm{Ch}\left(\operatorname{Mod}_{\text {cart }}(R)\right)$}

We will denote by $\mathrm{Ch}\left(\operatorname{Mod}_{\text {cart }}(R)\right)$ the category of unbounded chain complexes of $\operatorname{Mod}_{\text {cart }}(R)$.

We now recall some well-known facts of the category $\operatorname{Ch}(\mathcal{A})$ of unbounded chain complexes on an abelian category $\mathcal{A}$. A complex in $\mathcal{A}$,

$$
\cdots \stackrel{d_{n+2}}{\longrightarrow} X_{n+1} \stackrel{d_{n+1}}{\longrightarrow} X_{n} \stackrel{d_{n}}{\longrightarrow} X_{n-1} \stackrel{d_{n-1}}{\longrightarrow} \cdots
$$

will be denoted by $(X, d)$, or simply by $X$. And we will denote by $Z_{n} X=\operatorname{ker} d_{n}, K_{n} X=$ Coker $d_{n}$, $B_{n} X=\operatorname{Im} d_{n+1}$ and $H_{n} X=\frac{Z_{n} X}{B_{n} X}$, for every integer $n$. Given other complex $Y, \operatorname{Hom}(X, Y)$ will denote the complex defined by

$$
\operatorname{Hom}(X, Y)_{n}=\prod_{k \in \mathbb{Z}} \operatorname{Hom}_{R}\left(X_{k}, Y_{k+n}\right)
$$

and $\left((f) d_{n}^{H}\right)_{k}=f_{k} d_{k+n}^{Y}-(-1)^{n} d_{k}^{X} f_{k-1}$ for any $n \in \mathbb{Z}$. The class of all acyclic complexes will be denoted by $\mathcal{E}$.

Let us fix a cotorsion pair $(\mathcal{F}, \mathcal{C})$ in $\operatorname{Mod}_{\text {cart }}(R)$. We will consider the following subclasses of $\mathrm{Ch}\left(\operatorname{Mod}_{\mathrm{cart}}(R)\right)$ :

1. The class of $\mathcal{F}$-complexes, $\widetilde{\mathcal{F}}=\left\{X \in \mathcal{E}: Z_{n} X \in \mathcal{F}, \forall n \in \mathbb{Z}\right\}$. 
2. The class of $\mathcal{C}$-complexes, $\widetilde{\mathcal{C}}=\left\{X \in \mathcal{E}: Z_{n} X \in \mathcal{C}, \forall n \in \mathbb{Z}\right\}$.

3. The class of $d g-\mathcal{F}$ complexes,

$$
d g \widetilde{\mathcal{F}}=\left\{X \in \operatorname{Ch}\left(\operatorname{Mod}_{\text {cart }}(R)\right): X_{n} \in \mathcal{F} \forall n \in \mathbb{Z} \text { and } \operatorname{Hom}(X, C) \text { is exact } \forall C \in \widetilde{\mathcal{C}}\right\} .
$$

4. The class of $d g-\mathcal{C}$ complexes,

$$
d g \widetilde{\mathcal{C}}=\left\{X \in \operatorname{Ch}(\mathcal{A}): X_{n} \in \mathcal{C} \forall n \in \mathbb{Z} \text { and } \operatorname{Hom}(F, X) \text { is exact } \forall F \in \tilde{\mathcal{F}}\right\}
$$

We start with the following lemma. We recall that $\lambda$ is fixed as in Proposition 4.2 for $\operatorname{Mod}_{\text {cart }}(R)$.

Lemma 6.1. Let $\kappa$ be a regular infinite cardinal such that $\kappa \geq \lambda$. Let $N=\left(N^{n}\right), M=\left(M^{n}\right)$ be exact complexes such that $N \subseteq M$. For each $n \in \mathbb{Z}$, let $X_{n}$ be a $\kappa$-presentable cartesian $R$-submodule of $M^{n}$. Then there exists an exact complex $T=\left(T^{n}\right)$ such that $N \subseteq T \subseteq M$, and for each $n \in \mathbb{Z}, T^{n} \supseteq N^{n}+X_{n}$, and the object $T^{n} / N^{n}$ is $\kappa-$ presentable.

Proof. (I) First, consider the particular case of $N=0$. Let $Y_{0}^{n}=X_{n}+\delta^{n-1}\left(X_{n-1}\right)$. Then $\left(Y_{0}^{n}\right)$ is a subcomplex of $M$.

If $i<\omega$ and $Y_{i}^{n}$ is a $\kappa$-presentable cartesian $R$-submodule of $M^{n}$, put $Y_{i+1}^{n}=Y_{i}^{n}+D_{i}^{n}+\delta^{n-1}\left(D_{i}^{n-1}\right)$ where $D_{i}^{n}$ is a $\kappa$-presentable cartesian submodule of $M^{n}$ such that $\delta^{n}\left(D_{i}^{n}\right) \supseteq Z_{n+1} M \cap Y_{i}^{n+1}$. (Such $D_{i}^{n}$ exists by Proposition 4.2, since $Z_{n+1} M \cap Y_{i}^{n+1} \subseteq \operatorname{Ker}\left(\delta^{n+1}\right)=\operatorname{Im}\left(\delta^{n}\right)$.) Let $T^{n}=\bigcup_{i<\omega} Y_{i}^{n}$. Then $Z_{n+1} M \cap T^{n+1}=\bigcup_{i<\omega}\left(Z_{n+1} M \cap Y_{i}^{n+1}\right) \subseteq \bigcup_{i<\omega} \delta^{n}\left(Y_{i+1}^{n}\right) \subseteq \delta^{n}\left(T^{n}\right)$. It follows that $T=\left(T^{n}\right)$ is an exact subcomplex of $M$. By our assumption on $\kappa, T^{n}$ is $\kappa$-presentable.

(II) In general, let $\bar{M}=M / N$ and $\bar{X}_{n}=\left(X_{n}+N^{n}\right) / N^{n}$. By part (I), there is an exact complex $\bar{T}$ such that $\bar{T} \subseteq \bar{M}$, and for each $n \in \mathbb{Z}, \bar{T}^{n} \supseteq \bar{X}_{n}$, and the cartesian $R$-module $\bar{T}^{n}$ is $\kappa$-presentable. Then $\bar{T}=T / N$ for an exact subcomplex $N \subseteq T \subseteq M$, and $T$ clearly has the required properties.

We will also need the following Lemma whose proof is similar to the previous one, so we will omit it.

Lemma 6.2. Let $\kappa$ be a regular infinite cardinal such that $\kappa \geq \lambda$. Let $N=\left(N^{n}\right), M=\left(M^{n}\right)$ be complexes such that $N \subseteq M$. For each $n \in \mathbb{Z}$, let $X_{n}$ be a $\kappa$-presentable cartesian submodule of $M^{n}$. Then there 
exists a complex $T=\left(T^{n}\right)$ such that $N \subseteq T \subseteq M$, and for each $n \in \mathbb{Z}, T^{n} \supseteq N^{n}+X_{n}$, and the object $T^{n} / N^{n}$ is $\kappa$-presentable.

Theorem 6.3. Let $(\mathcal{F}, \mathcal{C})$ be a cotorsion pair cogenerated by a set in $\operatorname{Mod}_{\mathrm{cart}}(R)$ and such that $\mathcal{F}$ contains a generator of $\operatorname{Mod}_{\text {cart }}(R)$. Then the induced pairs $(\widetilde{\mathcal{F}}, d g \widetilde{\mathcal{C}})$ and $(d g \widetilde{\mathcal{F}}, \widetilde{\mathcal{C}})$ are complete cotorsion pairs.

Proof. By [Gil04, Corollary 3.8] we have induced cotorsion pairs $(\widetilde{\mathcal{F}}, d g \widetilde{\mathcal{C}})$ and $(d g \widetilde{\mathcal{F}}, \widetilde{\mathcal{C}})$. By Gil07, Proposition 3.8] and [Hov02, Corollary 6.6] the pair $(d g \widetilde{\mathcal{F}}, \widetilde{\mathcal{C}})$ is complete. Finally to see that the pair $(\widetilde{\mathcal{F}}, d g \widetilde{\mathcal{C}})$ is complete we will prove that each complex $C \in \widetilde{\mathcal{F}}$ is $\widetilde{\mathcal{F}}^{\kappa}$-filtered (for some $\kappa \geq \lambda$ ), so the cotorsion pair $(\widetilde{\mathcal{F}}, d g \widetilde{\mathcal{C}})$ will be cogenerated by a set. Then the completeness follows from Quillen's small object argument (see [Hov02, Corollary 6.6]).

Let $C=\left(M^{n}\right) \in \widetilde{\mathcal{F}}$. Then for each $n \in \mathbb{Z}, Z_{n} C \in \mathcal{F}$ and therefore $Z_{n} C$ has an $\mathcal{F}^{\kappa}$-filtration $\mathcal{O}_{n}=\left(M_{\alpha}^{n} \mid \alpha \leq \sigma_{n}\right)$. For each $n \in \mathbb{Z}, \alpha<\sigma_{n}$, consider a $\kappa$-presentable cartesian $R$-module $A_{\alpha}^{n}$ such that $M_{\alpha+1}^{n}=M_{\alpha}^{n}+A_{\alpha}^{n}$, and the corresponding family $\mathcal{H}_{n}$ as in Lemma 5.2. Since the complex $C$ is exact, the $\mathcal{F}^{\kappa}$-filtration $\mathcal{O}_{n+1}$ determines a canonical prolongation of $\mathcal{O}_{n}$ into a filtration $\mathcal{O}_{n}^{\prime}=\left(M_{\alpha}^{n} \mid \alpha \leq \tau_{n}\right)$ of $M^{n}$ where $\tau_{n}=\sigma_{n}+\sigma_{n+1}$ (the ordinal sum).

By definition, for each $\alpha \leq \sigma_{n+1}, \delta^{n}$ maps $M_{\sigma_{n}+\alpha}^{n}$ onto $M_{\alpha}^{n+1}$. So for each $\alpha<\sigma_{n+1}$ there is a $\kappa$-presentable cartesian submodule $A_{\sigma_{n}+\alpha}^{n}$ of $M_{\sigma_{n}+\alpha+1}^{n}$ such that $\delta^{n}\left(A_{\sigma_{n}+\alpha}^{n}\right)=A_{\alpha}^{n+1}$. Since for each $\sigma_{n} \leq \alpha<\tau_{n}$ we have $\operatorname{Ker}\left(\delta^{n}\right) \subseteq M_{\alpha}^{n}$, it follows that $M_{\alpha+1}^{n}=M_{\alpha}^{n}+A_{\alpha}^{n}$.

Let $\mathcal{H}_{n}^{\prime}$ be the family corresponding to $A_{\alpha}^{n}\left(\alpha<\tau_{n}\right)$ by Lemma 5.2. Since each closed subset of $\sigma_{n}$ is also closed when considered as a subset of $\tau_{n}$, we have $\mathcal{H}_{n} \subseteq \mathcal{H}_{n}^{\prime}$. Note that, by [Ekl77, Theorem 1.2], the class of $\mathcal{F}^{\kappa}$-filtered cartesian $R$-modules is contained in $\mathcal{F}$, so $\mathcal{H}_{n}^{\prime} \subseteq \mathcal{F}$ by condition (H3) of Lemma 5.2 .

Notice that $Z_{n} C=M_{\sigma_{n}}^{n}=\sum_{\alpha<\sigma_{n}} A_{\alpha}^{n}$. We claim that for each closed subset $S \subseteq \tau_{n}$, we have $Z_{n} C \cap \sum_{\alpha \in S} A_{\alpha}^{n}=\sum_{\alpha \in S \cap \sigma_{n}} A_{\alpha}^{n} \in \mathcal{H}_{n}$. To see this, we first show that $\sum_{\alpha<\sigma_{n}} A_{\alpha}^{n} \cap \sum_{\alpha \in S} A_{\alpha}^{n}=$ $\sum_{\alpha \in S \cap \sigma_{n}} A_{\alpha}^{n}$. The inclusion $\supseteq$ is clear, so consider $a \in\left(\sum_{\alpha<\sigma_{n}} A_{\alpha}^{n}\right) \cap \sum_{\alpha \in S} A_{\alpha}^{n}$. Then $a=a_{\alpha_{0}}+\cdots+a_{\alpha_{k}}$ where $\alpha_{i} \in S, a_{\alpha_{i}} \in A_{\alpha_{i}}^{n}$ for all $i \leq k$, and $\alpha_{i}>\alpha_{i+1}$ for all $i<k$. W.l.o.g., we can assume that $\alpha_{0}$ is minimal possible. If $\alpha_{0} \geq \sigma_{n}$, then $a_{\alpha_{0}}=a-a_{\alpha_{1}}-\cdots-a_{\alpha_{k}} \in\left(\sum_{\alpha<\alpha_{0}} A_{\alpha}^{n}\right) \cap A_{\alpha_{0}}^{n} \subseteq \sum_{\alpha \in S, \alpha<\alpha_{0}} A_{\alpha}^{n}$ as 
$\alpha_{0} \in S$ and $S$ is closed, in contradiction with the minimality of $\alpha_{0}$. Hence $\alpha_{0}<\sigma_{n}$, and $a \in \sum_{\alpha \in S \cap \sigma_{n}} A_{\alpha}^{n}$. So $Z_{n} C \cap \sum_{\alpha \in S} A_{\alpha}^{n}=\sum_{\alpha \in S \cap \sigma_{n}} A_{\alpha}^{n}$, and the latter cartesian $R$-module is in $\mathcal{H}_{n}$ because $S \cap \sigma_{n}$ is closed in $\sigma_{n}$. This proves our claim.

By induction on $\alpha$, we will construct an $\widetilde{\mathcal{F}}^{\kappa}$-filtration $\left(C_{\alpha} \mid \alpha \leq \sigma\right)$ of $C$ such that $C_{\alpha}=\left(N_{\alpha}^{n}\right)$, $Z_{n} C_{\alpha} \in \mathcal{H}_{n}$ and $N_{\alpha}^{n} \in \mathcal{H}_{n}^{\prime}$ for each $n \in \mathbb{Z}$.

First, $C_{0}=0$, and if $C_{\alpha}$ is defined and $C_{\alpha} \neq C$, then for each $n \in \mathbb{Z}$ we take a $\kappa$-presentable object $X_{n}$ such that $X_{n} \nsubseteq N_{\alpha}^{n}$ in case $N_{\alpha}^{n} \subsetneq M^{n}$ (this is possible by our assumption on $\kappa$ ), or $X_{n}=0$ if $M^{n}=N_{\alpha}^{n}$. If $M^{n}=N_{\alpha}^{n}$ for all $n \in \mathbb{Z}$, we let $\sigma=\alpha$ and finish our construction.

By Lemma 6.1 there exists an exact subcomplex $T=\left(T^{n}\right)$ of $C$ containing $C_{\alpha}$ such that for each $n \in \mathbb{Z}, T^{n} \supseteq N_{\alpha}^{n}+X_{n}$, and the cartesian $R$-module $T^{n} / N_{\alpha}^{n}$ is $\kappa$-presentable. Then $Y_{n}=T^{n}=N_{\alpha}^{n}+X_{n}^{\prime}$ for a $\kappa$-presentable cartesian $R$-submodule $X_{n}^{\prime}$ of $M^{n}$. By condition (H4) of Lemma 5.2 (for $N=N_{\alpha}^{n}$ and $X=X_{n}^{\prime}$ ), there exists a object $Y_{n}^{\prime}=P_{n}$ in $\mathcal{H}_{n}^{\prime}$ such that $N_{\alpha}^{n}+X_{n}^{\prime}=T^{n} \subseteq P_{n}$ and $P_{n} / N_{\alpha}^{n}$ is $\kappa$-presentable. Iterating this process we obtain a countable chain $Y_{n} \subseteq Y_{n}^{\prime} \subseteq Y_{n}^{\prime \prime} \subseteq \ldots$ whose union $N_{\alpha+1}^{n} \in \mathcal{H}_{n}^{\prime}$ by condition (H2) of Lemma 5.2. Then $C_{\alpha+1}=\left(N_{\alpha+1}^{n}\right)$ is an exact subcomplex of $C$ containing $C_{\alpha}$. Since $N_{\alpha+1}^{n} \in \mathcal{H}_{n}^{\prime}$, we have $Z_{n} C_{\alpha+1}=Z_{n} C \cap N_{\alpha+1}^{n} \in \mathcal{H}_{n}$ by the claim above.

In order to prove that $C_{\alpha+1} / C_{\alpha} \in \widetilde{\mathcal{F}}^{\kappa}$, it remains to show that for each $n \in \mathbb{Z}, Z_{n}\left(C_{\alpha+1} / C_{\alpha}\right) \in \mathcal{F}$. Since the complex $C_{\alpha+1} / C_{\alpha}$ is exact, it suffices to prove that $F=\left(\delta^{n}\left(N_{\alpha+1}^{n}\right)+N_{\alpha}^{n+1}\right) / N_{\alpha}^{n+1} \in \mathcal{F}$.

We have $N_{\alpha+1}^{n}=\sum_{\alpha \in S} A_{\alpha}^{n}$ where w.l.o.g., $S$ is a closed subset of $\tau_{n}$ containing $\sigma_{n}$. Let $S^{\prime}=\{\alpha<$ $\left.\sigma_{n+1} \mid \sigma_{n}+\alpha \in S\right\}$. Then $S^{\prime}$ is a closed subset on $\tau_{n+1}=\sigma_{n+1}+\sigma_{n+2}$. Indeed, for each $\alpha \in S^{\prime}$, we have

$$
\begin{gathered}
\sum_{\beta<\alpha} A_{\beta}^{n+1} \cap A_{\alpha}^{n+1}=\delta^{n}\left(\sum_{\beta<\sigma_{n}+\alpha} A_{\beta}^{n}\right) \cap \delta^{n}\left(A_{\sigma_{n}+\alpha}^{n}\right) \\
\subseteq \delta^{n}\left(\sum_{\beta<\sigma_{n}+\alpha, \beta \in S} A_{\beta}^{n}\right)=\sum_{\beta<\alpha, \beta \in S^{\prime}} A_{\alpha}^{n+1}
\end{gathered}
$$

where the inclusion $\subseteq$ holds because $S$ is closed in $\tau_{n}$ and $\operatorname{Ker}\left(\delta^{n}\right) \subseteq \sum_{\beta<\sigma_{n}+\alpha} A_{\beta}^{n}$.

Since $\delta^{n}\left(N_{\alpha+1}^{n}\right)=\sum_{\beta \in S^{\prime}} A_{\beta}^{n+1}$, and $N_{\alpha}^{n+1}=\sum_{\beta \in T} A_{\beta}^{n+1}$ for a closed subset $T$ of $\tau_{n+1}$, we have $F=\sum_{\beta \in S^{\prime} \cup T} A_{\beta}^{n+1} / \sum_{\beta \in T} A_{\beta}^{n+1}$, so $F \in \mathcal{F}$ by condition (H3) of Lemma 5.2 for $\mathcal{H}_{n+1}^{\prime}$. This finishes the proof of $C_{\alpha+1} / C_{\alpha} \in \widetilde{\mathcal{F}}^{\kappa}$. 
If $\alpha$ is a limit ordinal we define $C_{\alpha}=\bigcup_{\beta<\alpha} C_{\beta}=\left(N_{\alpha}^{n}\right)$. Then $N_{\alpha}^{n} \in \mathcal{H}_{n}^{\prime}$ by condition (H2) of Lemma 5.2, and $Z_{n} C_{\alpha}=Z_{n} C \cap N_{\alpha}^{n} \in \mathcal{H}_{n}$ by the claim above. This finishes the construction of the $\widetilde{\mathcal{F}}^{\kappa}$-filtration of $C$.

\section{$7 \quad$ Model category structures on $\mathrm{Ch}\left(\operatorname{Mod}_{\text {cart }}(R)\right)$}

In this section we will see that the induced cotorsion pairs in Theorem 6.3 give rise to an abelian model structure in $\mathrm{Ch}\left(\operatorname{Mod}_{\mathrm{cart}}(R)\right)$ in which the trivial objects are the exact complexes. According to Hovey in Hov02 we have to prove that we have induced complete cotorsion pairs of the form $(\mathcal{C}, \mathcal{D} \cap \mathcal{E})$ and $(\mathcal{C} \cap \mathcal{E}, \mathcal{D})$, where $\mathcal{C}$ and $\mathcal{D}$ are classes of complexes in $\mathrm{Ch}\left(\operatorname{Mod}_{\text {cart }}(R)\right)$. Following [Hov02, if we have two complete cotorsion pairs $\left(\mathcal{C}, \mathcal{C}^{\prime}\right)$ and $\left(\mathcal{D}^{\prime}, \mathcal{D}\right)$ in $\operatorname{Ch}\left(\operatorname{Mod}_{\text {cart }}(R)\right)$, we will say that they are compatible if $\mathcal{D}^{\prime}=\mathcal{C} \cap \mathcal{E}$ and $\mathcal{C}^{\prime}=\mathcal{D} \cap \mathcal{E}$.

Lemma 7.1. Let $(\mathcal{F}, \mathcal{C})$ be a cotorsion pair in $\operatorname{Mod}_{\text {cart }}(R)$ cogenerated by a set, such that $\mathcal{F}$ contains a generator of $\operatorname{Mod}_{\text {cart }}(R)$ and $\mathcal{F}$ is closed under taking kernels of epimorphisms. Then the pairs $(\widetilde{\mathcal{F}}, d g \widetilde{\mathcal{C}})$ and $(d g \widetilde{\mathcal{F}}, \widetilde{\mathcal{C}})$ are compatible.

Proof. According to [Gil07, Corollary 3.9(3)] we have just to check that

$$
\operatorname{Ext}_{M o d_{\text {cart }}(R)}^{n}(F, C)=0
$$

for any $n>0$ and any $F \in \mathcal{F}$ and $C \in \mathcal{C}$. By definition of cotorsion pair, $\operatorname{Ext}_{M o d_{\text {cart }}(R)}^{1}(F, C)=0$. Given any exact sequence

$$
0 \rightarrow C \rightarrow M \rightarrow N \rightarrow F \rightarrow 0
$$

representing an element in $\operatorname{Ext}_{M o d_{\text {cart }}(R)}^{2}(F, C)$, we can construct the sequence

$$
0 \rightarrow C \rightarrow P \rightarrow G \rightarrow F \rightarrow 0
$$

which represents the same element in $\operatorname{Ext}_{M d_{\text {cart }}(R)}^{2}(F, C)$ but with $G \in \mathcal{F}$ (since $\mathcal{F}$ contains a generator of $\left.\operatorname{Mod}_{\text {cart }}(R)\right)$ and $Z=\operatorname{ker}(G \rightarrow F) \in \mathcal{F}$ (because $\mathcal{F}$ is closed under kernels of epimorphisms). So 
$\operatorname{Ext}_{M o d_{\text {cart }}(R)}^{1}(Z, C)=0$, and therefore $0 \rightarrow C \rightarrow P \rightarrow Z \rightarrow 0$ splits. But this means that $0 \rightarrow C \rightarrow$ $P \rightarrow G \rightarrow F \rightarrow 0$ (and therefore also $0 \rightarrow C \rightarrow M \rightarrow N \rightarrow F \rightarrow 0$ ) represents the zero element in $\operatorname{Ext}_{M o d_{\text {cart }}(R)}^{2}(F, C)$. Proceeding inductively in this way we get our claim.

Theorem 7.2. Let $(\mathcal{F}, \mathcal{C})$ be a cotorsion pair in $\operatorname{Mod}_{\text {cart }}(R)$ cogenerated by a set and such that $\mathcal{F}$ contains a generator of $\operatorname{Mod}_{\mathrm{cart}}(R)$ and $\mathcal{F}$ is closed under kernels of epimorphisms. The compatible cotorsion pairs $(d g \widetilde{\mathcal{F}}, \widetilde{\mathcal{C}})$ and $(\widetilde{\mathcal{F}}, d g \widetilde{\mathcal{C}})$ induce an abelian model category structure in $\operatorname{Ch}\left(\operatorname{Mod}_{\text {cart }}(R)\right)$. In this abelian model structure the weak equivalences are the homology isomorphisms, cofibrations (resp. trivial cofibrations) are monomorphisms whose cokernels are in $d g \widetilde{\mathcal{F}}$, (resp. trivial cofibrations are monomorphisms with cokerneles in $\widetilde{\mathcal{F}}$ ) and fibrations (resp. trivial fibrations) are epimorphisms whose kernels are in $d g \widetilde{\mathcal{C}}$ (resp. trivial fibrations are epimorphisms with kernels in $\widetilde{\mathcal{C}}$ ). The corresponding homotopy category to this model structure is $\mathbf{D}\left(\operatorname{Mod}_{\text {cart }}(R)\right)$, the derived category of $\operatorname{Mod}_{\text {cart }}(R)$.

Proof. The proof of both theorems is a consequence of Theorem 6.3, Lemma 17.1 and Hov02, Theorem $2.2]$.

\section{The Derived category of quasi-coherent sheaves on an Artin stack}

Throughout this section by an algebraic stack we mean an Artin stack with separated and quasi-compact diagonal in the sense of [LMB00].

An algebraic stack is geometric if it is quasi-compact with affine diagonal (this definition is due to Toën and Vezzosi in [TV08 and Lurie [Lur05]). For geometric stacks, Gross in his thesis Gro10, (3.5.5)Theorem] proves in a very elegant way that $\mathfrak{Q} \mathfrak{c o}(\mathcal{X})$ admits enough flat objects (that is, every quasi-coherent $\mathcal{O}_{\mathcal{X}}$-module is a quotient of a flat quasi-coherent $\mathcal{O}_{\mathcal{X}}$-module. The corresponding result for schemes was known from ATJLL97 by using the derived category of quasi-coherent sheaves. We recall that if $\mathcal{F}$ denotes the class of flat quasi-coherent $\mathcal{O}_{\mathcal{X}}$-modules, then the elements in $\mathcal{F}^{\perp}$ are known as cotorsion quasi-coherent $\mathcal{O}_{\mathcal{X}}$-modules. 
Theorem 8.1. Let $\mathcal{X}$ be an algebraic stack with enough flats (for instance a geometric stack). There is a monoidal model category structure on $\mathrm{Ch}(\mathfrak{Q} \mathfrak{c o}(\mathcal{X}))$ where weak equivalences are homology isomorphisms, the cofibrations (resp. trivial cofibrations) are the monomorphisms whose cokernels are dg-flat complexes (res. flat complexes). The fibrations (resp. trivial fibrations) are the epimorphisms whose kernels are dg-cotorsion complexes (resp. cotorsion complexes). The associated homotopy category is $\mathbf{D}(\mathfrak{Q} \mathfrak{c o}(\mathcal{X}))$, the derived category of $\mathfrak{Q} \mathfrak{c o}(\mathcal{X})$.

Proof. Let $\mathcal{C}$ be the category of affine schemes smooth over $\mathcal{X}$ and $R$ the sheaf of rings $\mathcal{O}_{\mathcal{X}}$. We will apply Theorem 7.2 to the class $\mathcal{F}$ of flat cartesian $R$-modules. The fact that $\left(\mathcal{F}, \mathcal{F}^{\perp}\right)$ is a cotorsion pair can be easily derived from Proposition 4.2 and the small object argument [Hov98, Theorem 2.1.14]. Namely, by EE05, Theorem 4.1] for each $M \in \operatorname{Mod}_{\text {cart }}(R)$ there exists a short exact sequence $0 \rightarrow C \rightarrow F \rightarrow M \rightarrow 0$ with $C \in \mathcal{F}^{\perp}$ and $F \in \mathcal{F}$. Now if $M \in^{\perp}\left(\mathcal{F}^{\perp}\right)$ the sequence splits and so $M$ is also flat cartesian $R$-module. Hence $\left(\mathcal{F}, \mathcal{F}^{\perp}\right)$ is a cotorsion pair. By Proposition 4.2 we see that each $F \in \mathcal{F}$ is $\mathcal{F}^{\kappa}$-filtered for certain $\kappa \geq \lambda$. So $\mathcal{F}$ is cogenerated by the set of iso classes of $\kappa$-presentable objects in $\mathcal{F}$.

Finally to get that the model structure is monoidal we apply [Gil07, Theorem 5.1] by observing that the class $\mathcal{F}$ satisfies conditions (1), (2) and (3) of that Theorem.

For the next application we need to recall the definition of (infinite dimensional) vector bundle (cf.

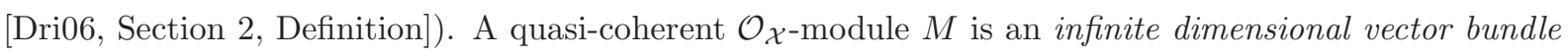
if it is locally projective. Let $\mathcal{F}$ be the class of all infinite dimensional vector bundles. An algebraic stack $\mathcal{X}$ has the resolution property if every quasi-coherent sheaf is a quotient of a filtered direct limit of locally free sheaves of finite type. In particular if an algebraic stack satisfies the resolution property, the class $\mathcal{F}$ contains a family of generators for $\mathfrak{Q} \mathfrak{c o}(\mathcal{X})$, in other words $\mathfrak{Q} \mathfrak{c o}(\mathcal{X})$ has enough vector bundles. By [Tot04, Theorem 1.1] for a normal noetherian algebraic stack $\mathcal{X}$ the resolution property is equivalent to $\mathcal{X}$ being isomorphic to the quotient stack of some quasi-affine scheme by an action of the group $\mathrm{GL}_{n}$. This has been extended by Gro10, Theorem 6.3.1] for non-normal noetherian stacks, and by Ryd13 without noetherian hypothesis. 
Theorem 8.2. Let $\mathcal{X}$ be an algebraic stack with pointwise affine stabilizer group that satisfies the resolution property (for instance if $\mathcal{X}$ is a global quotient stack, cf. Tho87, 2.18]). There is a monoidal model category structure on $\operatorname{Ch}(\mathfrak{Q c o}(\mathcal{X}))$ where weak equivalences are homology isomorphisms, the cofibrations (resp. trivial cofibrations) are the monomorphisms whose cokernels are dg-complexes of (infinite dimensional) vector bundles (resp. exact complexes of vector bundles whose every quasicoherent sheaf of cycles is a vector bundle). The fibrations (resp. trivial fibrations) are the epimorphisms whose kernels are dg-orthogonal to the class of infinite dimensional vector bundles. The associated homotopy category is $\mathbf{D}(\mathfrak{Q} \mathfrak{c o}(\mathcal{X}))$, the derived category of $\mathfrak{Q} \mathfrak{c o}(\mathcal{X})$.

Proof. Again we let $\mathcal{C}$ be the category of affine schemes smooth over $\mathcal{X}$ and $R$ the sheaf of rings $\mathcal{O}_{\mathcal{X}}$ Then we will apply Theorem 7.2 to the class $\mathcal{F}$ of locally projective cartesian $R$-modules. To show that $\left(\mathcal{F}, \mathcal{F}^{\perp}\right)$ is a cotorsion pair cogenerated by a set we will first prove that every $F \in \mathcal{F}$ is $\mathcal{F}^{\kappa}$-filtered for certain $\kappa \geq \lambda$. By Kaplansky Theorem each $F(v)$ is a direct sum of countably generated projective $R(v)$ modules, in particular every $F(v)$ possess a filtration $\mathcal{P}_{v}$ by countably generated projective $R(v)$-modules. Let $\mathcal{H}_{v}$ be the family associated to $\mathcal{P}_{v}$ by Lemma 5.2 and $\left\{x_{v, \alpha} \mid \alpha<\tau_{v}\right\}$ be an $R(v)$-generating set of the $R(v)$-module $F(v)$. W.l.o.g., we can assume that for some ordinal $\tau, \tau=\tau_{v}$ for all $v$.

Let us denote by $\left(F_{\alpha} \mid \alpha \leq \tau\right)$ the desired $\mathcal{F}^{\kappa}$-filtration that we will get for $F$. Let $F_{0}=0$. Assume that $F_{\alpha}$ is defined for some $\alpha<\tau$ such that $F_{\alpha}(v) \in \mathcal{H}_{v}$ and $x_{v, \beta} \in F_{\alpha}(v)$ for all $\beta<\alpha$ and all $v$. Set $N_{v, 0}=F_{\alpha}(v)$. By condition (H4) of Lemma 5.2, there is a module $N_{v, 1} \in \mathcal{H}_{v}$ such that $N_{v, 0} \subseteq N_{v, 1}$ and $N_{v, 1} / N_{v, 0}$ is $\lambda$-presentable.

By Proposition 4.2 there is a cartesian $R$-submodule $G_{1}$ of $F$ such that $F_{\alpha} \subseteq G_{1}$ and $G_{1} / F_{\alpha}$ is $\lambda$ presentable. Therefore $G_{1}(v)=N_{v, 1}+\left\langle S_{v}\right\rangle$ for a set $S_{v} \subseteq G_{1}(v)$, of cardinality $<\lambda$. Now by condition (H4) of Lemma 5.2 there is a module $N_{v, 2} \in \mathcal{H}_{v}$ such that $G_{1}(v)=N_{v, 1}+\left\langle S_{v}\right\rangle \subseteq N_{v, 2}$ and $N_{v, 2} / N_{v, 1}$ is $\lambda$ presentable. Following in this manner, we get a countable chain $\left(G_{n} \mid n<\aleph_{0}\right)$ of cartesian $R$-submodules of $F$ and a countable chain $\left(N_{v, n} \mid n<\aleph_{0}\right)$ of $R(v)$-submodules of $F(v)$. We define $F_{\alpha+1}=\sum_{n<\aleph_{0}} G_{n}$. Then it is clear that $F_{\alpha+1} \subseteq F$ is a cartesian $R$-module satisfying $F_{\alpha+1}(v)=\sum_{n<\aleph_{0}} G_{n}(v)$ for each $v$. By condition (H2) of Lemma 5.2, we deduce that $F_{\alpha+1}(v) \in \mathcal{H}_{v}$ and $\left|F_{\alpha+1}(v) / F_{\alpha}(v)\right|<\lambda$. Therefore 
$F_{\alpha+1} / F_{\alpha} \in \mathcal{F}^{\kappa}$. Finally if $\alpha \leq \tau$ is an ordinal limit and $F_{\beta}$ is defined for every $\beta<\alpha$, we define $F_{\alpha}=\sum_{\beta<\alpha} F_{\beta}$. Since $x_{v, \alpha} \in F_{\alpha+1}(v)$ for each $v$ and $\alpha<\tau$, we have $F_{\tau}(v)=F(v)$. So $\left(F_{\alpha} \mid \alpha \leq \tau\right)$ is the desired $\mathcal{F}^{\kappa}$-filtration of $F$. Then, by [Ekl77, Theorem 1.2] the pair $\left(\mathcal{F}, \mathcal{F}^{\perp}\right)$ is cogenerated by the set of iso classes of $\mathcal{F}^{\kappa}$ cartesian $R$-modules.

By [EEGRO04, Lemma 2.4, Theorem 2.5], for all $A \in \operatorname{Mod}_{\text {cart }}(R)$ there exists a short exact sequence

$$
0 \rightarrow A \rightarrow P \rightarrow Z \rightarrow 0
$$

where $P \in \mathcal{F}^{\perp}$ and $Z$ has an $\mathcal{F}$-filtration. Given any $M \in \operatorname{Mod}_{\text {cart }}(R)$, since $\mathcal{X}$ satisfies the resolution property, there exists a short exact sequence

$$
0 \rightarrow U \rightarrow G \rightarrow M \rightarrow 0
$$

where $G$ is a direct sum of locally free cartesian $R$-modules (of finite type). Now let

$$
0 \rightarrow U \rightarrow N \rightarrow Z \rightarrow 0
$$

be exact with $N \in \mathcal{F}^{\perp}$ and $Z$ admitting an $\mathcal{F}$-filtration. Form a pushout and get

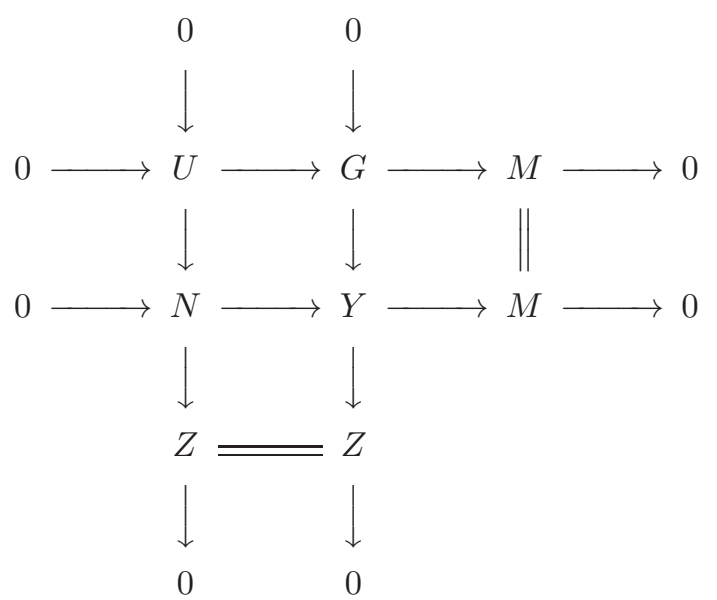

Then since $G \in \mathcal{F}$ and $Z$ has an $\mathcal{F}$-filtration (so $Z \in \mathcal{F}$, since $\mathcal{F}$ is closed under $\mathcal{F}$-filtrations), we see that $Y \in \mathcal{F}$. Also $N \in \mathcal{F}^{\perp}$. Hence if $M \in^{\perp}\left(\mathcal{F}^{\perp}\right)$ we get that $0 \rightarrow N \rightarrow Y \rightarrow M \rightarrow 0$ splits and so $M$ is a direct summand of $Y \in \mathcal{F}$. But then $M \in \mathcal{F}$ because $\mathcal{F}$ is closed under direct summands. 
Again, the model structure is monoidal because the class of locally projective cartesian $R$-modules is contained in the class of flat cartesian $R$-modules, hence condition (1) of [Gil07, Theorem 5.1] holds (and so in particular $\mathcal{F}$ contains the unit $R$ for the monoidal structure on $\mathfrak{Q} \mathfrak{c o}(\mathcal{X})$, so condition (3) holds). It is also immediate to notice that the tensor product of two locally projective cartesian $R$-modules is again locally projective. So condition (2) of [Gil07, Theorem 5.1] is satisfied, what finishes the proof.

\section{Remarks:}

1. There is a slightly different notion of algebraic stack in the literature, due to Goerss Goe04 by considering that the diagonal morphism is affine and that there is an affine scheme $U$ and a faithfully flat 1-morphism, $p: U \rightarrow \mathcal{X}$. Given a flat Hopf algebroid in [Nau7, Section 3] it is shown that there is an equivalence between the category of (left) comodules on a flat Hopf algebroid and the category of $\mathcal{O}_{\mathcal{X}}$-modules on a certain algebraic stack, in the sense of Goerss (see $\underline{\text { Holl08 }}$ for a generalization). The results of this paper apply to this setting, just by picking the suitable category $\operatorname{Mod}_{\text {cart }}(R)$, thus providing monoidal model category structures on $\mathrm{Ch}(\Gamma)$, the category of unbounded complexes of (left) $\Gamma$-comodules over $(A, \Gamma)$. In case of "well-behaved" flat Hopf algebroids (see Hov04 for a precise formulation of "well-behaved") Hovey in Hov04 already defined and studied a model category structure over $\mathrm{Ch}(\Gamma)$. Its associated model category is the derived category of $(A, \Gamma)$.

2. Let $X$ be a quasi-compact semi-separated scheme. The derived category of quasi-coherent sheaves is a stable homotopy category in the sense of HPS97. This was shown in AJPV08. The main ingredients of their proof where the facts that $\mathfrak{Q} \mathfrak{c o}(X)$ is a Grothendieck category (for any scheme $X$ ) and that each quasi-coherent $\mathcal{O}_{X}$-module admits flat resolutions provided that $X$ is quasicompact and semi-separated. For the category $\mathfrak{Q} \mathfrak{c o}(\mathcal{X})(\mathcal{X}$ an arbitrary Artin stack) the axioms (a) and (d) of [HPS97, Definition 1.1.4] trivially hold. By Corollary $4.7 \mathfrak{Q} \mathfrak{c o}(\mathcal{X})$ is Grothendieck, hence each cohomology functor on $\mathfrak{Q} \mathfrak{c o}(\mathcal{X})$ is representable (cf. ATJLSS00, Theorem 5.8]), so the axiom (e) of HPS97 holds. Now it seems reasonable to conjecture that using the results of this 
paper and [Gro10, (3.5.5) Theorem] the remainder axioms (b) and (c) of [HPS97, Definition 1.1.4] may be followed as in AJPV08 for the case of (quasi-compact and semi-separated) schemes.

3. Let Flat $\mathcal{X}$ be the class of flat quasi-coherent $\mathcal{O}_{X}$-modules over a geometric stack $\mathcal{X}$. Again by Gro10, (3.5.5) Theorem] and the application of the small object argument (cf. EE05, Theorem 4.1]) the pair (Flat $\mathcal{X}$, Flat $\mathcal{X}^{\perp}$ ) is a complete cotorsion pair.

In his thesis Mur08, Murfet defines the mock homotopy category of projectives, $\mathbf{K}_{m}(\operatorname{Proj} X)$ for a quasi-compact and semi-separated scheme $X$. The starting point is Neeman's description (cf. [Nee08, Facts 2.14(iii)]) on the affine case $X=\operatorname{Spec}(R)$ of $\mathbf{K}(\operatorname{Proj} X)$ as the Verdier quotient

$$
\mathbf{K}(R \text {-Flat }) / \mathbf{K}(\widetilde{R \text {-Flat }})
$$

and define $\mathbf{K}_{m}(\operatorname{Proj} X)$ as the corresponding Verdier quotient $\mathbf{K}($ Flat $X) / \mathbf{K}(\widetilde{\text { Flat } X})$ for arbitrary quasi-compact and semi-separated schemes (see [Mur08, Definition 3.3 and Proposition 3.4]). Then he proves the existence of a right adjoint functor of the Verdier quotient map $j^{*}: \mathbf{K}($ Flat $X) \rightarrow$ $\mathbf{K}_{m}(\operatorname{Proj} X)$, thus extending to quasi-compact and semi-separated schemes the affine case [Nee10, Theorem 0.1]. Now it is a general fact that any complete cotorsion pair $(\mathcal{A}, \mathcal{B})$ in $\operatorname{Ch}\left(\operatorname{Mod}_{\text {cart }}(R)\right)$ enables to show the existence of right (resp. left) adjoints of the embeddings $\mathbf{K}(\mathcal{A}) \rightarrow \mathbf{K}\left(\operatorname{Mod}_{\text {cart }}(R)\right)$ and $\mathbf{K}(\mathcal{B}) \rightarrow \mathbf{K}\left(\operatorname{Mod}_{\text {cart }}(R)\right)$, provided that $\mathcal{A}$ is closed under taking suspensions (see EBIJR12, Theorem 3.5]). Now by Theorem 6.3 the pair (Flat $\mathcal{X}$, Flat $\left.\mathcal{X}^{\perp}\right)$ in $\mathfrak{Q} \mathfrak{c o}(\mathcal{X})$ gives rise to the complete cotorsion pair $(\widetilde{\mathcal{F}}, d g \widetilde{\mathcal{C}})$ in $\operatorname{Ch}(\mathfrak{Q} \mathfrak{c o}(\mathcal{X}))\left(\right.$ where $\mathcal{F}=$ Flat $\mathcal{X}$ and $\mathcal{C}=$ Flat $\left.\mathcal{X}^{\perp}\right)$. Hence we can extend Murfet and Neeman results to geometric stacks, to conclude that for a geometric stack $\mathcal{X}$ the canonical map $j^{*}: \mathbf{K}($ Flat $\mathcal{X}) \rightarrow \mathbf{K}_{m}(\operatorname{Proj} \mathcal{X})$ has a right adjoint functor. As a consequence there is a localization sequence

$$
\mathbf{K}(\widetilde{\text { Flat } \mathcal{X}}) \rightarrow \mathbf{K}(\text { Flat } \mathcal{X}) \rightarrow \mathbf{K}_{m}(\operatorname{Proj} \mathcal{X}) .
$$

\section{References}

[ATJLl97] L. Alonso Tarrío, A. Jeremías López, J. Lipman, Local homology and cohomology on 
schemes, Ann. Sci. École Norm. Sup. 30(4) (1997), 1-39.

[AJPV08] L. Alonso Tarrío, A. Jeremías López, M. Pérez Rodríguez and M. J. Vale GonSALves, The derived category of quasi-coherent sheaves and axiomatic stable homotopy, Adv. in Math. 218(2008), 1224-1252.

[ATJlsS00] L. Alonso Tarrío, A. Jeremías López and M. J. Souto Salorio, Localization in categories of complexes and unbounded resolutions, Canad. J. Math. 52(2000), 225-247.

[EBIJR12] D. Bravo, E. Enochs, A. Iacob, O. Jenda and J. Rada, Cotorsion pairs in C(R-Mod), Rocky Mountain J. Math. 42 (2012), 1787-1802.

[Con00] B. Conrad, Grothendieck duality and base change, Lecture Notes in Mathematics, 1750, Springer-Verlag, 2000.

[Dri06] V. Drinfeld, Infinite-dimensional vector bundles in algebraic geometry: an introduction, in 'The Unity of Mathematics', Birkh\&quot; auser, Boston 2006, pp. 263-304.

[Ekl77] P.C. EkLOF, Homological algebra and set theory, Trans. Amer. Math. Soc. 227(1977), 207-225.

[EE05] E. Enochs And S. Estrada, Relative homological algebra in the category of quasi-coherent sheaves, Adv. in Math. 194(2005), 284-295.

[EE12] E. Enochs AND S. Estrada, Cartesian modules in small categories, preprint, arXiv:math.AG/1203.5724.

[EeGro04] E. Enochs, S. Estrada, J.R. García Rozas, and L. Oyonarte, Flat covers in the category of quasi-coherent sheaves over the projective line. Comm. Algebra. 32(2004), 1497-1508.

[EGPt12] S. Estrada, P. Guil Asensio, M. Prest and J. Trlifaj, Model category structures arising from Drinfeld vector bundles, Adv. in Math. 231(2012), 1417-1438.

[Gil04] J. Gillespie, The flat model structure on Ch(R), Trans. Amer. Math. Soc. 356(2004), 33693390. 
[Gil07] J. Gillespie, Kaplansky classes and derived categories, Math. Z. 257(2007), 811-843.

[Gil08] J. GilLESPIE, Cotorsion pairs and degreewise homological model structures, Homol. Homot. App. 10(2008), 283-304.

[GT06] R. Göbel and J. Trlifaj, Approximations and Endomorphism Algebras of Modules, GEM 41, W. de Gruyter, Berlin 2006.

[Goe04] P. Goerss, (Pre-)sheaves of ring spectra over the moduli stack of formal group laws. Axiomatic, Enriched and Motivic Homotopy Theory, 101-131, NATO Sci. Ser. II Math. Phys. Chem., 131, Kluwer Acad. Publ., Dordrecht, 2004.

[Gro10] P. Gross, Vector bundles as generators on schemes and stacks, PhD. Thesis, Düsseldorf, May 2010.

[Holl08] S. Hollander, Characterizing algebraic stacks, Proc. Amer. Math. Soc. 136 (2008), 1465-1476.

[Hov98] M. Hovey, Model Categories, MSM 63, Amer. Math. Soc., Providence, RI, 1998.

[Hov01] M. Hovey, Model category structures on chain complexes of sheaves, Trans. Amer. Math. Soc. 353(6)(2001), 2441-2457.

[Hov02] M. HoveY, Cotorsion pairs, model category structures, and representation theory, Math. Z. 241 (2002), 553-592.

[Hov04] M. Hovey, Homotopy theory of comodules over a Hopf algebroid, Homotopy theory: relations with algebraic geometry, group cohomology, and algebraic K-theory, 261?304, Contemp. Math., 346, Amer. Math. Soc., Providence, RI, 2004.

[HPS97] M. Hovey, J. H. Palmieri and N. P. Strickland, Axiomatic stable homotopy theory, Mem. Amer. Math. Soc.128(1997), no. 610.

[LMB00] G. Laumon and L. Moret-Bailly, Homology, Springer-Verlag, Berlin, 2000. 
[Lur05] J. LuRIE, Tannaka duality for geometric stacks, preprint, arXiv:math.AG/0412266v2.

[May01] J. P. MaY, The additivity of traces in triangulated categories. Adv. in Math. 163(2001), 34-73.

[Mur08] D. Murfet, The Mock Homotopy Category of Projectives and Grothendieck Duality, PhD thesis, Aust. National U. 2008.

[Nau7] N. NAumann, The stack of formal groups in stable homotopy theory. Adv. in Math. 215(2007), $569-600$.

[Nee08] A. NeEman, The homotopy category of flat modules, and Grothendieck duality, Invent. Math. 174(2008), 255-308.

[Nee10] A. Neeman, Some adjoints in homotopy categories, Ann. Math. 171(2010), 2143-2155.

[Qui67] D. Quillen, Homotopical Algebra, LNM 43, Springer-Verlag, Berlin/New York, 1967.

[Ols07] M. Olsson, Sheaves on Artin stacks. J. Reine Angew. Math. 603(2007), 55-112.

[RG71] M. Raynaud and L. Gruson, Critères de platitude et de projectivité, Invent. Math. 13(1971), $1-89$.

[Ryd13] D. RYDH, Noetherian approximation of algebraic spaces and stacks, preprint, arXiv: math. AG/0904.0227v3.

[Tho87] R. W. Thomason, Equivariant resolutions, linearization, and Hilbert's fourteenth problem over arbitrary base schemes. Adv. in Math.65(1987), 16-34.

[TV08] B. Tö̈n And G. Vezzosi, Homotopical algebraic geometry. II. Geometric stacks and applications., Mem. Amer. Math. Soc.193(2008), no. 902.

[Tot04] B. TотAro, The resolution property for schemes and stacks, J. reine angew. Math. 577 (2004), $1-22$. 